(C) 2008 The Japan Society of Applied Physics

\title{
Highly Birefringent Index-Guiding Photonic Crystal Fiber with Squeezed Differently Sized Air-Holes in Cladding
}

\author{
Yuh-Sien Sun, Yuan-Fong ChAU*, Han-Hsuan YeH, and Din Ping TsaI ${ }^{1}$ \\ Department of Electronic Engineering, Ching Yun University, Jung-Li 320, Taiwan, R.O.C. \\ ${ }^{1}$ Department of Physics and Center for Nanostorage Research, National Taiwan University, Taipei 106, Taiwan, R.O.C.
}

(Received November 6, 2007; accepted January 27, 2008; published online May 16, 2008)

We propose a novel high-birefringence index-guiding photonic crystal fiber (PCF). This PCF is composed of a solid silica core and a cladding with two differently sized squeezed elliptical air-holes. The mode birefringence of a fundamental mode in such PCFs is analyzed numerically by the finite-element method. Numerical results reveal that an extraordinarily high modal birefringence at the excitation wavelength of $\lambda=1550 \mathrm{~nm}, 2.6 \times 10^{-2}$, is acquired. The contributions of the cladding with two different sizes of air-hole ellipticity, the center-to-center distance between the air-holes, and the the number of cladding rings as well as the confinement loss to the birefringence are systematically evaluated. The evolution of birefringence with the structural variations shows that our highly birefringent fiber can be designed in a controlled manner.

[DOI: 10.1143/JJAP.47.3755]

KEYWORDS: birefringence, photonic crystal fibers, finite-element method

\section{Introduction}

In recent years, photonic crystal fibers (PCFs), optical fibers that guide light in a defect surrounded by a regular lattice of air-holes, have attracted considerable interest as an alternative to conventional optical fibers for manipulating the properties of light and its applications. ${ }^{1-5)}$ Because of the design flexibility and high index contrast, highly birefringent (Hi-Bi) PCFs can be easily realized. Hi-Bi single-mode fibers allow light to propagated in the preferred polarization direction. One possible use of Hi-Bi PCFs is as polarizationmaintaining fibers (PMFs), which can eliminate the influence of polarization mode dispersion or stabilize the operation of optical devices. In recent years, different approaches for achieving high birefringence have been explored for PCFs. Hi-Bi index guiding PCFs with holes of different diameters along the two orthogonal fiber axes or with asymmetric core designs have been proposed. ${ }^{6-10)}$ Very recently, a tremendous $\mathrm{Hi}-\mathrm{Bi}$ polarization-maintaining $\mathrm{PCF}^{4)}$ was proposed, and its numerical results showed group birefringence of $3.78 \times 10^{-2}$. Because the gaps between the holes are very narrow, the corresponding fiber structure with optimal structural parameters is weak under external forces. This implies that the unit size of air-holes in the PCF cladding has the drawback of narrow gaps between air-holes, where by sections overlap, making the structure physically unfeasible.

On the Basis of our previous studies, ${ }^{11,12)}$ we propose a new structure of the high-birefringence index-guiding PCF. This PCF is composed of a solid silica core and a PC cladding with squeezed elliptical air-holes of different distances between air-holes along $x$ - and $y$-axes. The proposed structure has elliptical air-holes of two different sizes to replace the singular size of elliptical air-holes in a PCF cladding, and is different from that proposed in conventional PCF designs. ${ }^{1-10)}$ Owing to the gaps between two different air-holes being larger than between of single holes, the corresponding fiber structure designed with our proposed complex air-holes in a PCF cladding is stronger than that with a singular size of air-holes in conventional

*E-mail address: yfc01@cyu.edu.tw
PCF designs under external forces. The birefringence of our proposed structure is the result of the whole cladding asymmetry. The fiber core is a point defect, which is formed by the omission of one smaller elliptical air-hole in the center of this structure; thus, it is possible to create a highbirefringence PCF. Microstructured optical fibers with elliptical air-holes were experimentally realized in 2004. ${ }^{13)}$ With the appearance of new methods for fabricating microstructured fibers, such as preform drilling, sol-gel casting, and tapering, ${ }^{13-15)}$ it is possible for us to draw PCFs with such a structure. In this paper, the origin of the birefringence is discussed and its dependence on the structural parameters is analyzed in the proposed PCFs with different sizes of elliptical air-holes in PCF cladding. We also discuss the effect of the rings of air-holes and confinement loss on of birefringence.

\section{Simulation Method}

Among the full vectorial methods used in modeling PCFs, ${ }^{16-20)}$ the finite-element method (FEM) is particularly effective for handling curved interfaces with high accuracy and obviously is a good choice for combined elliptical shape and waveguide analysis. The numerical method used in this study is FEM, which is adequate for the analysis of general dielectric waveguide geometries. It has already been successfully applied to investigate dispersion properties of triangular and cobweb PCFs. ${ }^{21)}$ The fiber cross section representation is very accurate as the domain is divided into subdomains of triangular or quadrilateral shape with which refractive index profiles can be properly represented. As described in refs. 22 and 23, the Maxwell equations with a magnetic field formulation can be presented as

$$
\nabla \times\left(\varepsilon_{\mathrm{r}}^{-1} \nabla \times h\right)-k_{0}^{2} \mu_{\mathrm{r}} h=0,
$$

where $h$ is the magnetic field, $\epsilon_{\mathrm{r}}$ and $\mu_{\mathrm{r}}$ are the relative dielectric permittivity and magnetic permeability complex tensors, respectively, and $k_{0}=2 \pi / \lambda$, is the wave number in the vacuum, $\lambda$ being the wavelength. The magnetic field of the modal solution is expressed as $h=H e^{-\gamma z}$, where $H$ is the field distribution on the transverse plane and $\gamma=\alpha+j \beta$ is the complex propagation constant with $\alpha$ being the attenuation constant and $\beta$ the phase constant. With the application 
of variational finite-element procedure, the above full vector equation yields the following algebraic problem: ${ }^{22,23)}$

$$
\mathbf{A}-\left(\frac{\gamma}{k_{0}}\right)^{2} \mathbf{B H}=0,
$$

where the eigenvector $\mathbf{H}$ and the eigenvalue $\left(\gamma / k_{0}\right)^{2}$ provide, respectively, the full vectorial magnetic field distribution and the effective index of the mode. In the present formulation, triangular high-order edge elements are used. In order to model infinite PCF with two-dimensional finite geometry (i.e., to enclose the computational domain without affecting the numerical solution), it is necessary to use anisotropic perfectly matched layers (PMLs) that are placed before the outer boundary. This formulation enable us to deal with anisotropic material in terms of both dielectric permittivity and magnetic permeability, and allows anisotropic PMLs to be directly implemented.

\section{Models, Results, and Discussion}

The cross section of our proposed PCF model is shown in Fig. 1. We use two different sizes of elliptical air-holes in PCF cladding, where each lattice point forms the cladding with two different sizes of elliptical air-holes with pitch (center-to-center distance between the holes) $\Lambda_{x}$ and $\Lambda_{y}$ along the $x$-axis and the $y$-axis, respectively. As seen in Fig. $1, d_{1}$ and $d_{2}$ denote the length of the large elliptical airholes along $x$ - (minor axes) and $y$ - (major axis) directions, respectively. In the same manner, $d_{3}$ and $d_{4}$ represent the small elliptical air-holes. The core is formed by omitting of one small elliptical air-hole in the center of this structure. The refractive index of the background silica is set as $n=$ 1.45 and that of the air-holes is set to be 1 throughout the simulations. The structure is derived from a triangularlatticed PC compressed in the $x$-direction. The structural

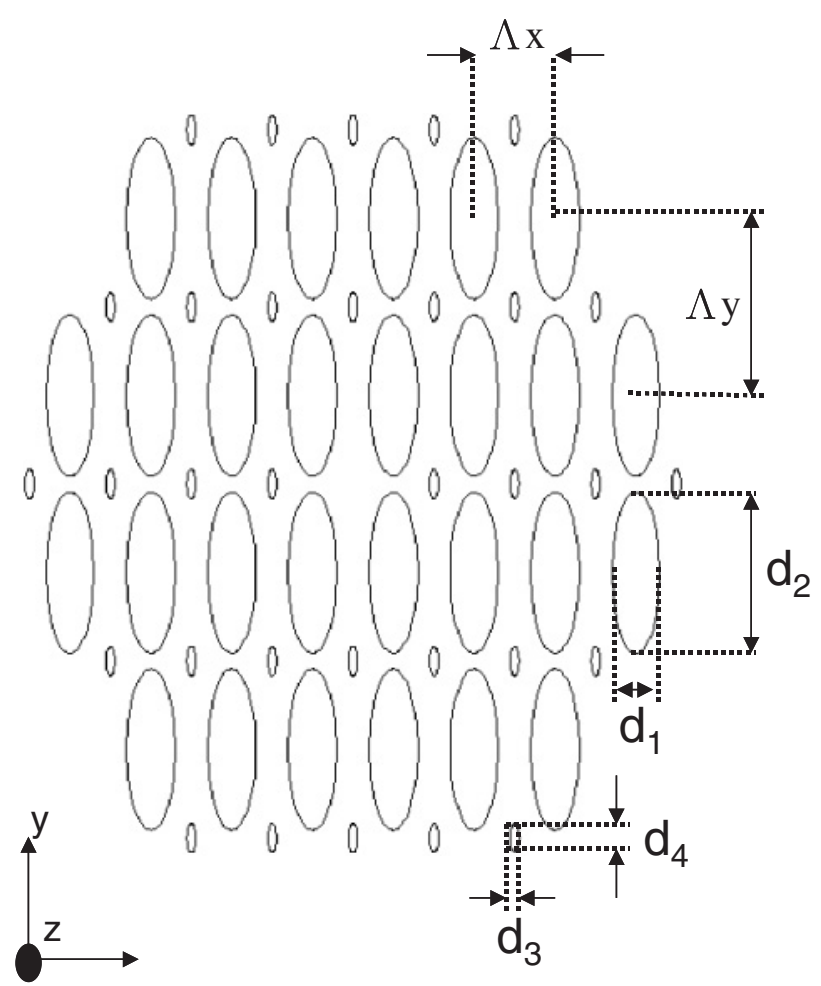

Fig. 1. Cross section of our proposed birefringent PCF. compression can be done during fiber drawing, following a technique described in ref. 24.

In Fig. 1, birefringence is introduced owing to the difference between the two orthogonal directions. In the $x$ and $y$-directions of two different linear, homogeneous, and isotropic dielectrics, Maxwell's equations impose the boundary conditions for normal and the transverse components of magnetic fields. There is one important fact, that is, the mode fields in PCFs resemble the $\mathrm{HE}_{11}$ mode in step-index fibers, and we refer to them as $\mathrm{HE}_{11 x}$ and $\mathrm{HE}_{11 y}$. The doublet components of the fundamental mode, as we know, are degenerate in conventional standard fibers and in circular air-holes with triangular lattice PCFs. However, when the air-holes are elliptical, the degeneracy splits significantly. Regardless of whether the modes are confined or leaky, the birefringence will be defined as $\Delta n=\left|n_{\text {eff }}^{x}-n_{\text {eff }}^{y}\right|$, where $n_{\mathrm{eff}}^{x}$ and $n_{\mathrm{eff}}^{y}$ are the refractive indices of the $x$ - and $y$ polarization modes, respectively. To illustrate the field profile of our proposed PCFs, the fundamental mode of our designed fiber with the parameters $\Lambda_{x}=2.2 \mu \mathrm{m}, \Lambda_{y}=$ $6.4 \mu \mathrm{m}, d_{1}=1.8 \mu \mathrm{m}, d_{2}=6 \mu \mathrm{m}, d_{3}=0.328 \mu \mathrm{m}, d_{4}=1.1$ $\mu \mathrm{m}, \eta\left(d_{1} / d_{3}\right)=5.5$, and elliptical ratio $d_{1} / d_{2}=d_{3} / d_{4}=0.3$ $\left(d_{1} / d_{2}\right.$ for big holes, and $d_{3} / d_{4}$ for small holes, see Fig. 1$)$ at excitation wavelength $\lambda=1550 \mathrm{~nm}$, as shown in Fig. 2, illustrates the confinement of light in PCF. The reason why we choose the elliptical ratio is that we found the simulation results of modal birefringence to be very similar to the increase in the elliptical ratio the range of $[2.5,3.6]$. Thus, we choose the elliptical ratio to be 0.3 as an example. It can be seen in Fig. 2 that the $x$-polarized mode is stronger than the of $y$-polarized mode owing to the $x$-polarized states having a lower air filling fraction than $y$-polarized states. The asymmetry due to two different sizes of air-holes in the PCF cladding is one of the key factors in determining the localization extent of the transverse mode. The simulation results for the $x$ - and $y$-polarized modes are strongly

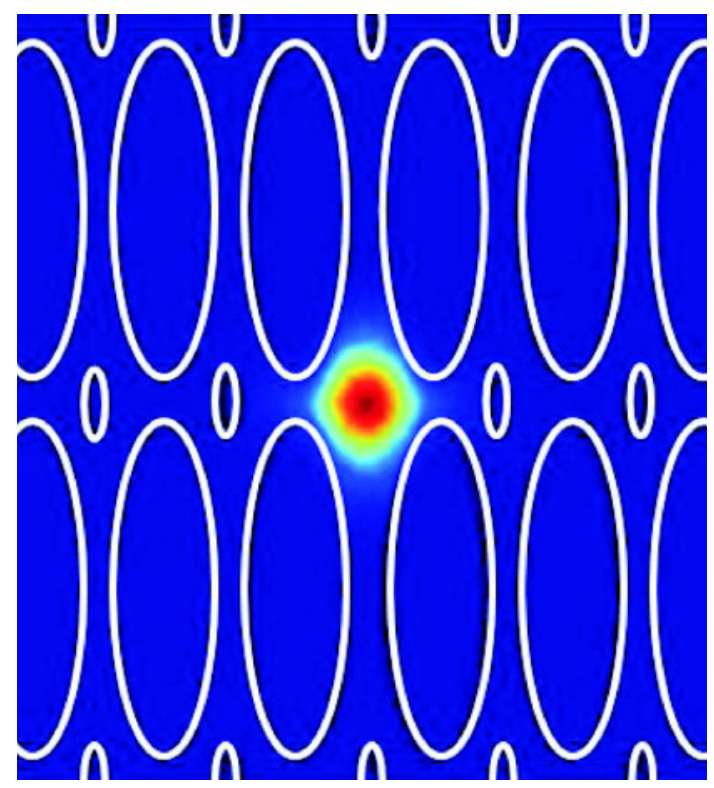

Fig. 2. (Color online) Mode field pattern with $\Lambda_{x}=2.2 \mu \mathrm{m}, \Lambda_{y}=6.4$ $\mu \mathrm{m}, d_{1}=1.8 \mu \mathrm{m}, d_{2}=6 \mu \mathrm{m}, d_{3}=0.328 \mu \mathrm{m}, d_{4}=1.1 \mu \mathrm{m}, \eta\left(d_{1} / d_{3}\right)=$ 5.5 , and elliptical ratio $d_{1} / d_{2}=d_{3} / d_{4}=0.3$ at excitation wavelength $\lambda=1550 \mathrm{~nm}$. 


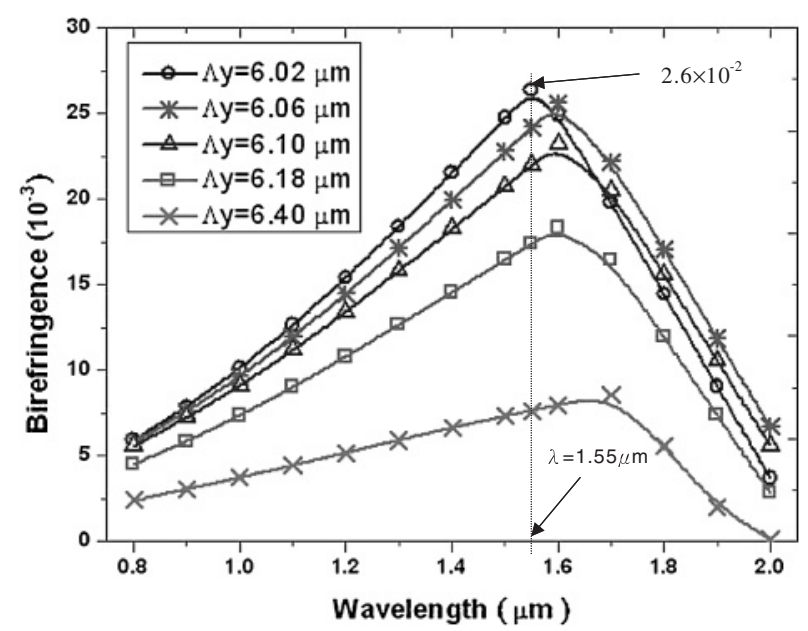

Fig. 3. Birefringence of the proposed PCF as a function of wavelength in the range from $\lambda=0.8$ to $2.0 \mu \mathrm{m}$ for $\Lambda_{y}=6.02,6.06,6.10,6.18$, and $6.40 \mu \mathrm{m}$ with fixed $\Lambda_{x}=1.82 \mu \mathrm{m}, d_{1}=1.8 \mu \mathrm{m}, d_{2}=6 \mu \mathrm{m}, d_{3}=0.328$ $\mu \mathrm{m}, d_{4}=1.1 \mu \mathrm{m}, \eta\left(d_{1} / d_{3}\right)=5.5$, and elliptical ratio $d_{1} / d_{2}=d_{3} / d_{4}=$ 0.3 .

bounded in the high index core region with the effective indices $n_{\text {eff }}^{x}=1.3382547$ and $n_{\text {eff }}^{y}=1.37901$, respectively, giving the birefringence $\Delta n=\left|n_{\mathrm{eff}}^{x}-n_{\mathrm{eff}}^{y}\right|=1.1637 \times$ $10^{-2}$, which is much higher than those obtained from conventional step-index fiber $\left(5 \times 10^{-4}\right),{ }^{6}$ circular air-holes $\left(3.7 \times 10^{-3}\right)^{3)}$ and elliptical hollow PCFs $\left(2.35 \times 10^{-3}\right){ }^{24)}$ Simulation results show that the effect of the two different air-hole sizes in the PCF cladding can significantly enhance the birefringence.

The effective index and birefringence of PCFs are related to the wavelength. As expected, the difference between two polarized direction modes in the PC cladding asymmetry can cause high birefringence in PCFs. Figure 3 shows the birefringence of the proposed PCF as a function of wavelength in the range from $\lambda=0.8$ to $2.0 \mu \mathrm{m}$, with fixed $\Lambda_{x}=1.82 \mu \mathrm{m}, \quad d_{1}=1.8 \mu \mathrm{m}, \quad d_{2}=6 \mu \mathrm{m}, \quad d_{3}=0.328 \mu \mathrm{m}$, $d_{4}=1.1 \mu \mathrm{m}, \eta\left(d_{1} / d_{3}\right)=5.5$, and elliptical ratio $d_{1} / d_{2}=$ $d_{3} / d_{4}=0.3$ and different values of $\Lambda_{y}$. It is clear that the birefringence is sensitive to the air-hole spacing $\Lambda_{y}$ with fixed $\Lambda_{x}$ as wavelength $\lambda$ increases in the range of $[0.8,1.8]$ $\mu \mathrm{m}$. The tendency of the birefringence curve in Fig. 3 is the same as those in the case of the elliptical-hole PCFs ${ }^{25,26)}$ and a squeezed hexagonal lattice $\mathrm{PCF}^{4}$ ) with increasing lattice pitch along the $x$-axis as wavelength $\lambda$ increases in the range of $[0.8,1.6] \mu \mathrm{m}$. We obtain the birefringence curves for $\Lambda_{y}=6.02,6.06,6.10,6.18$, and $6.40 \mu \mathrm{m}$.

As shown in Fig. 3, the family of curves of birefringence shift upward, corresponding to a decrement in the $\Lambda_{y}$ value. Namely, the air-hole spacing is also one of the key factors in determining the localization extent of the transverse mode. The dispersion curves of birefringence increase with the wavelength in the range of $[0.8,1.6] \mu \mathrm{m}$. when $\Lambda_{y}$ is squeezed (for example, $\Lambda_{y}=6.02,6.06$, and $6.10 \mu \mathrm{m}$ ), while it decreases when $\Lambda_{y}$ is enlarged (for example, $\Lambda_{y}=$ 6.18 and $6.4 \mu \mathrm{m}$ ), because squeezing $\Lambda_{y}$ further increases the difference between the effective indices of $y$ - and $x$-polarization modes, while enlarging $\Lambda_{y}$ reduces the difference. To some extent, this characteristic is similar to that of conventional elliptical core fibers. ${ }^{27-29)}$ The corresponding wavelength $\lambda=1.55 \mu \mathrm{m}$ with birefringence reaches its maximum

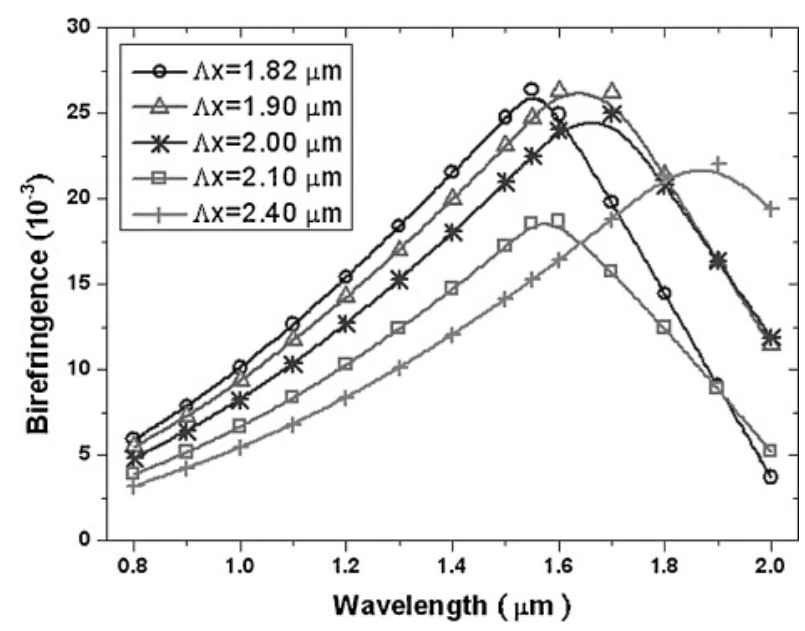

Fig. 4. Birefringence of the proposed PCF as a function of wavelength in the range from $\lambda=0.8$ to $2.0 \mu \mathrm{m}$ for $\Lambda_{x}=1.82,1.9,2.0,2.1$, and $2.4 \mu \mathrm{m}$ with fixed $\Lambda_{y}=6.02 \mu \mathrm{m}, d_{1}=1.8 \mu \mathrm{m}, d_{2}=6 \mu \mathrm{m}, d_{3}=0.328 \mu \mathrm{m}, d_{4}=$ $1.1 \mu \mathrm{m}, \eta\left(d_{1} / d_{3}\right)=5.5$, and elliptical ratio $d_{1} / d_{2}=d_{3} / d_{4}=0.3$.

value $\Delta n=2.6 \times 10^{-2}$ at $\Lambda_{y}=6.02 \mu \mathrm{m}$ and its minimum value $\Delta n=7.5 \times 10^{-3}$ at $\Lambda_{y}=6.4 \mu \mathrm{m}$, resulting in an enhancement of $1.85 \times 10^{-2}$ via the effect of scaling the airhole spacing along the $y$-axis. It may then be concluded that the birefringence can be increased by squeezing the hole spacing $\Lambda_{y}$ along the $y$-axis.

We further studied the effect of squeezing the elliptical air-holes along the other direction ( $x$-axis) on the birefringence of PCFs. Figure 4 illustrates the birefringence of the proposed PCF as a function of wavelength in the range from $\lambda=0.8$ to $2.0 \mu \mathrm{m}$ for $\Lambda_{x}=1.82,1.9,2.0,2.1$, and $2.4 \mu \mathrm{m}$ with fixed $\Lambda_{y}=6.02 \mu \mathrm{m}, d_{1}=1.8 \mu \mathrm{m}, d_{2}=6 \mu \mathrm{m}, d_{3}=$ $0.328 \mu \mathrm{m}, d_{4}=1.1 \mu \mathrm{m}, \eta\left(d_{1} / d_{3}\right)=5.5$, and elliptical ratio $d_{1} / d_{2}=d_{3} / d_{4}=0.3$. These curves also increase monotonically with the increase of wavelength. However, compared with Fig. 3, the curves in Fig. 4 show less difference in birefringence, i.e., the values of birefringence varies from $\Delta n=1.527 \times 10^{-2}$ to $2.627 \times 10^{-2}$ at excitation wavelength $\lambda=1.55 \mu \mathrm{m}$. It should be noted here that the birefringence corresponding to $\Lambda_{x}$ in the range of $[1.82,2.4] \mu \mathrm{m}$ is higher than the magnitude of $10^{-2}$, indicating that the proposed structure is robust against manufacturing inaccuracies.

The field confinement and its decay rate depend on the airhole diameter, their pitch, $\Lambda_{x}$ or $\Lambda_{y}$, and on the number of rings. In practice, 6 to 10 rings of air-holes are often needed to reduce the confinement loss to an acceptable level. For the sake of accuracy, we use 10 rings in this work. Figure 5 shows the birefringence of the proposed PCF as a function of PC cladding ring number, with fixed $\Lambda_{x}=1.82 \mu \mathrm{m}$, $d_{1}=1.8 \mu \mathrm{m}, \quad d_{2}=6 \mu \mathrm{m}, \quad d_{3}=0.328 \mu \mathrm{m}, \quad d_{4}=1.1 \mu \mathrm{m}$, $\eta\left(d_{1} / d_{3}\right)=5.5$, and elliptical ratio $d_{1} / d_{2}=d_{3} / d_{4}=0.3$ and different values of $\Lambda_{y}$. The air-hole spacing along the $y$-axis, $\Lambda_{y}$, is varied in the range of $[6.02,6.18] \mu \mathrm{m}$. The influence of $\Lambda_{y}$ in the PCF cladding on the birefringence stability is also significant, as illustrated in Fig. 5. As can be expected from Figs. 3 and 4, the smaller $\Lambda_{y}$, the higher the birefringence obtained. The reason why the birefringence with small $\Lambda_{y}$ is higher than those with large $\Lambda_{y}$ is related to the contribution of the average refractive index, which gives 


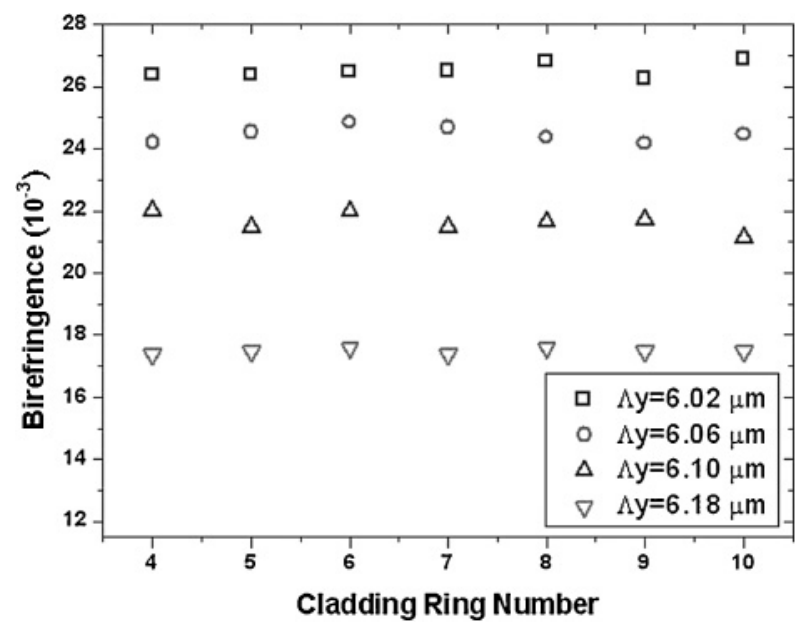

Fig. 5. Birefringence of the proposed PCF as a function of PC cladding ring number and with fixed $\Lambda_{x}=1.82 \mu \mathrm{m}, d_{1}=1.8 \mu \mathrm{m}, d_{2}=6 \mu \mathrm{m}, d_{3}=$ $0.328 \mu \mathrm{m}, d_{4}=1.1 \mu \mathrm{m}, \eta\left(d_{1} / d_{3}\right)=5.5$, elliptical ratio $d_{1} / d_{2}=d_{3} / d_{4}=$ 0.3 and different values of $\Lambda_{y}$. The air-hole spacing along the $y$-axis, $\Lambda_{y}$, was varied in the range of $[6.02,6.18] \mu \mathrm{m}$.

rise to more fields confined in the core region; thus the birefringence is more stable even in the case of 10 rings.

FEM with PMLs, which are placed before the outer boundary can be used to calculate the confinement loss of PCFs. The confinement loss of a guided mode in PCFs can be determined according to the following formulation:

$$
\begin{aligned}
\text { Confinement loss }= & \frac{2 \times 10^{10}}{\ln 10} \frac{2 \pi}{\lambda} \operatorname{Im}\left[n_{\mathrm{eff}}^{i}\right] \mathrm{dBm} / \mathrm{km}, \\
& (i=x, y),
\end{aligned}
$$

where $\operatorname{Im}\left[n_{\text {eff }}^{i}\right]$ is the imaginary part of the effective index of the guided mode.

The imaginary part of the complex effective index represents the loss. Figure 6 shows the confinement loss as a function of wavelength in the range from $\lambda=0.8$ to 2.0 for $\Lambda_{y}=6.02,6.10$, and $6.40 \mu \mathrm{m}$ with fixed $\Lambda_{x}=1.82 \mu \mathrm{m}$, $d_{1}=1.8 \mu \mathrm{m}, \quad d_{2}=6 \mu \mathrm{m}, \quad d_{3}=0.328 \mu \mathrm{m}, \quad d_{4}=1.1 \mu \mathrm{m}$, $\eta\left(d_{1} / d_{3}\right)=5.5$, and elliptical ratio $d_{1} / d_{2}=d_{3} / d_{4}=0.3$. It can be seen in Fig. 6 that the confinement losses at different $\Lambda_{y}$ are $42.16 \mathrm{~dB} / \mathrm{km}$ for $\Lambda_{y}=6.40,0.682 \mathrm{~dB} / \mathrm{km}$ for $\Lambda_{y}=6.10$, and $0.363 \mathrm{~dB} / \mathrm{km}$ for $\Lambda_{y}=6.02$ at the wavelength of $\lambda=1.55 \mu \mathrm{m}$. This shows that the effect of $\Lambda_{y}$ on the optical loss (mode confinement) is appreciable and the confinement loss decreases as the distance between air-holes along the $y$-axis $\left(\Lambda_{y}\right)$ decreases. For the case of large $\Lambda_{y}$ (e.g., $\Lambda_{y}=6.40$ ), the confinement loss is large owing to the greater distance between air-holes along the $y$-axis, whereby more field in the core region of PCF conner be confined. Contrary to the case of small $\Lambda_{y}$ (e.g., $\Lambda_{y}=6.10$ and $\Lambda_{y}=$ 6.02), a shorter distance between air-holes along the $y$-axis can confine more field in the core region of PCF.

Now we vary $\Lambda_{x}$ to investigate its effect on confinement loss. Figure 7 shows the confinement loss as a function of wavelength in the range from $\lambda=0.8$ to 2.0 for $\Lambda_{x}=1.82$, 2.00 , and $2.40 \mu \mathrm{m}$ with fixed $\Lambda_{y}=6.02 \mu \mathrm{m}, d_{1}=1.8 \mu \mathrm{m}$, $d_{2}=6 \mu \mathrm{m}, \quad d_{3}=0.328 \mu \mathrm{m}, \quad d_{4}=1.1 \mu \mathrm{m}, \quad \eta\left(d_{1} / d_{3}\right)=5.5$, and elliptical ratio $d_{1} / d_{2}=d_{3} / d_{4}=0.3$. In this case, the confinement losses are $0.363 \mathrm{~dB} / \mathrm{km}$ for $\Lambda_{x}=1.82,0.023$ $\mathrm{dB} / \mathrm{km}$ for $\Lambda_{x}=2.00$, and $6.306 \times 10^{-4} \mathrm{~dB} / \mathrm{km}$ for $\Lambda_{x}=$

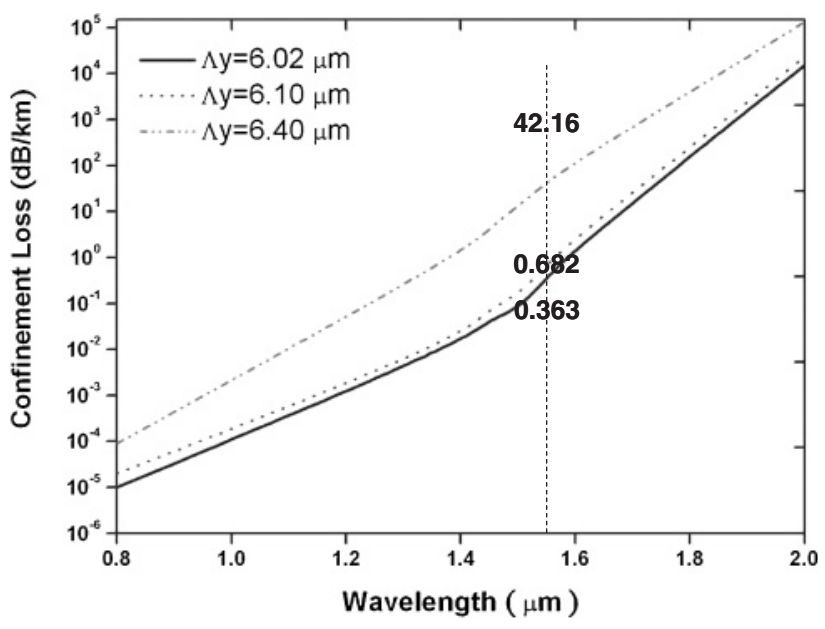

Fig. 6. The confinement loss as a function of wavelength in the range from $\lambda=0.8$ to 2.0 for $\Lambda_{y}=6.02,6.10$, and $6.40 \mu \mathrm{m}$ with fixed $\Lambda_{x}=1.82 \mu \mathrm{m}, d_{1}=1.8 \mu \mathrm{m}, d_{2}=6 \mu \mathrm{m}, d_{3}=0.328 \mu \mathrm{m}, d_{4}=1.1 \mu \mathrm{m}$, $\eta\left(d_{1} / d_{3}\right)=5.5$, and elliptical ratio $d_{1} / d_{2}=d_{3} / d_{4}=0.3$.

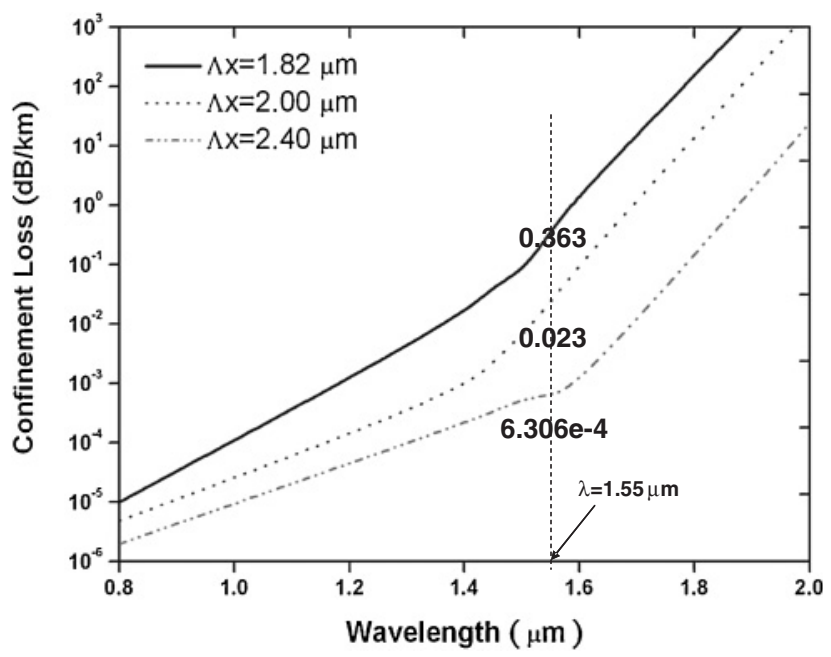

Fig. 7. The confinement loss as a function of wavelength in the range from $\lambda=0.8$ to 2.0 for $\Lambda_{x}=1.82,2.00$, and $2.40 \mu \mathrm{m}$ with fixed $\Lambda_{y}=6.02 \mu \mathrm{m}, d_{1}=1.8 \mu \mathrm{m}, d_{2}=6 \mu \mathrm{m}, d_{3}=0.328 \mu \mathrm{m}, d_{4}=1.1 \mu \mathrm{m}$, $\eta\left(d_{1} / d_{3}\right)=5.5$, and elliptical ratio $d_{1} / d_{2}=d_{3} / d_{4}=0.3$.

2.40 at the wavelength of $\lambda=1.55 \mu \mathrm{m}$. Unlike the case shown in Fig. 6 [varying distance between air-holes along the $y$-axis $\left(\Lambda_{y}\right)$ ], it can be observed that the confinement loss increases as the distance between air-hole along the $x$-axis $\left(\Lambda_{x}\right)$ decreases. This is because a smaller stress factor makes the hole diameter smaller in the $x$-direction and thus allows greater leakage of the $y$-polarized field into the cladding than the $x$-polarized field. On the basis of this observation, the magnitude of confinement loss can be controlled by adjusting the values of $\Lambda_{y}$ and $\Lambda_{x}$. A large $\Lambda_{y}$ and a small $\Lambda_{x}$ will increase the confinement loss, as can be seen from the comparison of Figs. 6 and 7. This is because a smaller stress factor makes the hole diameter smaller in the $x$-direction and thus allows greater $y$-polarized field leakage into the cladding than $x$-polarized fields leakage. This may be desirable if one wants to reduce the required fiber length for achieving a certain extinction ratio according to the desired application. 
Similar to the conventional and elliptical hollow highbirefringence fibers, the merits of our proposed PCF discussed here can be advantageous for a number of applications $^{30-34)}$ and allow for highly birefringent devices with much broader wavelength ranges to be created. In addition, the high-birefringence EHPCFs, coupled with the use of endless single-mode PCFs or endless single-mode polarization-maintaining PCFs as lead-in/lead-out fibers, would allow for extremely broadband devices that cannot be created with conventional technologies.

\section{Conclusions}

A highly birefringent index-guiding squeezed elliptical air-holes PCF with different sizes of air-holes in the cladding is successfully demonstrated numerically by the finiteelement method. The high-birefringence is achieved by inducing air-holes sizes of different into a triangular structure. The two different sizes of squeezed elliptical air-holes in the PCF cladding could endow a new degree of freedom in the design of the birefringence in optical waveguides. The mode birefringence of the fundamental modes in such PCFs is analyzed by utilizing the intrinsically large index contrast in PCFs in combination with two different sizes of air-holes in the cladding. We have found that it is possible to create modal birefringence of at least two orders of magnitude higher than that of conventional Hi-Bi fibers ${ }^{6)}$ and one order of magnitude higher than those reported for other PCF structures. ${ }^{3,24)}$ The suggested structure possesses a large birefringence to separate the two polarization modes and is robust against manufacturing inaccuracies. Our simulation results provide valuable insight for the realization of PCFs with even higher birefringence than previously demonstrated.

\section{Acknowledgements}

The authors are grateful for the financial support from the National Science Council, Taiwan, R.O.C., under grant number NSC 96-2112-M-231-001-(1/3)-MY3 and NSC962120-M-002-017.

1) J. C. Knight, T. A. Birks, P. St. J. Russell, and D. M. Atkin: Opt. Lett. 21 (1996) 1547.

2) T. A. Birks, J. C. Knight, and P. St. J. Russell: Opt. Lett. 22 (1997) 961.

3) A. Ortigosa-Blanch, J. C. Knight, W. J. Wadsworth, J. Arriaga, B. J.
Mangan, T. A. Birks, and P. St. J. Russell: Opt. Lett. 25 (2000) 1325.

4) Y. Yue, G. Kai, Z. Wang, T. Sun, L. Jin, Y. Lu, C. Zhang, J. Liu, Y. Li, Y. Liu, S. Yuan, and X. Dong: Opt. Lett. 32 (2007) 469.

5) X. Yu, M. Yan, and P. Shum: IEEE Photonics Technol. Lett. 18 (2006) 1243.

6) J. Noda, K. Okamoto, and Y. Sasaki: J. Lightwave Technol. 4 (1986) 1071.

7) M. J. Steel and R. M. Osgood: J. Lightwave Technol. 19 (2001) 495.

8) K. Saitoh and M. Koshiba: IEEE Photonics Technol. Lett. 14 (2002) 1291.

9) A. Hochman and Y. Leviatan: Opt. Express 13 (2005) 6193.

10) F. Zhang, X. Liu, M. Zhang, and P. Ye: Chin. Opt. Lett. 5 (1997) 260.

11) Y.-S. Sun, Y.-F. Chau, H.-H. Yeh, T.-J. Yang, and D. P. Tsai: Appl. Opt. 46 (2007) 5276.

12) Y.-F. Chau, H.-H. Yeh, and D. P. Tsai: Jpn. J. Appl. Phys. 46 (2007) L1048.

13) N. A. Issa, M. A. van Eijkelenborg, M. Fellew, F. Cox, G. Henry, and M. C. J. Large: Opt. Lett. 29 (2004) 1336.

14) R. T. Bise and D. Trevor: Tech. Dig. Optical Fiber Communication Conf./Expo. Natl. Fiber Optic Engineers Conf., 2005, paper OWL6 (CD).

15) P. Domachuk, A. Chapman, E. Magi, M. J. Steel, H. C. Nguyen, and B. J. Eggleton: Appl. Opt. 44 (2005) 3885.

16) A. Ferrando, E. Silvestre, J. J. Miret, and P. Andres: Opt. Lett. 24 (1999) 276.

17) D. Mogilevtsev, T. A. Birks, and P. St. J. Russell: J. Lightwave Technol. 17 (1999) 2078.

18) Z. Zhu and T. G. Brown: Opt. Commun. 206 (2002) 333.

19) Z. Zhu and T. Brown: Opt. Express 10 (2002) 853.

20) F. Brechet, J. Marcou, D. Pagnoux, and P. Roy: Opt. Fiber Technol. 6 (2000) 181

21) A. Cucinotta, S. Selleri, L. Vincetti, and M. Zoboli: IEEE Photonics Technol. Lett. 14 (2002) 1530.

22) S. Selleri, L. Vincetti, A. Cucinotta, and M. Zoboli: Opt. Quantum Electron. 33 (2001) 359.

23) D. Ferrarini, L. Vincetti, M. Zoboli, A. Cucinotta, and S. Selleri: Opt. Express 10 (2002) 1314.

24) Y. Jung, S. R. Han, Soan Kim, U. C. Paek, and K. Oh: Opt. Lett. 31 (2006) 2681.

25) M. J. Steel and R. M. Osgood, Jr.: Opt. Lett. 26 (2001) 229.

26) M. J. Steel and R. M. Osgood: J. Lightwave Technol. 19 (2001) 495.

27) B. Y. Kim, J. N. Blake, S. Y. Huang, and H. J. Shaw: Opt. Lett. 12 (1987) 729.

28) J. N. Blake, S. Y. Huang, B. Y. Kim, and H. J. Shaw: Opt. Lett. 12 (1987) 732.

29) S. Y. Huang, J. N. Blake, and B. Y. Kim: J. Lightwave Technol. 8 (1990) 23.

30) J. N. Blake, B. Y. Kim, and H. J. Shaw: Opt. Lett. 11 (1986) 177.

31) W. V. Sorin, B. Y. Kim, and H. J. Shaw: Opt. Lett. 11 (1986) 581.

32) A. M. Vengsarkar, W. C. Michie, L. Jankovic, B. Culshaw, and R. O. Claus: J. Lightwave Technol. 12 (1994) 170.

33) S. H. Yun, I. K. Hwang, and B. Y. Kim: Opt. Lett. 21 (1996) 27.

34) H. S. Park, K. Y. Song, S. H. Yun, and B. Y. Kim: J. Lightwave Technol. 20 (2002) 1864. 